\title{
66 Endurance training and tumor immunology
}

The physiological fundamentals known to date suggest that endurance training likely has a positive influence on lowering the risk of malignant diseases. Facts favoring this likelihood include that moderate physical exertion is associated with enhanced mobilization of natural killer cells, their overall higher cytotoxic capacity as well as the phagocytosis capability of granulocytes that is improved under these conditions ( $\downarrow$ Chapter 62 ). This enables our immune system to attack and eliminate the constant flux of neoplastic cells occurring in our body.

Breast cancer in women is one example of regular physical activity leading to a lower disease incidence and reduced mortality risk. This was shown by the results of 3 large-scaled studies: conducted by the California Teachers Study on more than 130,000, the NIH-AARP Diet and Health study on close to 183,000 and the Nurses' Health Study on over 95,000 participants (Peters et al. 2009, West-Wright et al. 2009, Eliassen et al. 2010). The strongest protection is conferred when physical activity is started at a young age (Maruti et al. 2008, Leitzmann et al. 2008). But even one hour of walking a day reduces the risk of cancer (Hildebrand et al. 2013).

Besides estradiol, insulin also plays a role in the development of breast cancer. This hormone can accelerate the growth of tumor cells. Because overweight persons often have insulin resistance ( Chapter 40) and larger concentrations of unbound insulin circulate in their bodies for a period of time, insulin is also a co-factor in the higher rate of breast cancer in obese women. The observed lower frequency of this type of cancer in athletic women is additionally attributable to the weight loss and associated reduction in insulin resistance.

Due to the fact that insulin levels are reduced by endurance training, the risk for bowel cancer is also lower (Wolin et al. 2011). In this context, the authors of this large meta-analysis describe the specific mechanism to be the lower development of adenomas and their slower conversion to malignant carcinoma.

Nevertheless, global protection against malignant tumors by merely strengthening endurance capacity is not tenable given the numerous noxae, developmental mechanisms and, not least, the multitude of individual genetic predispositions that exist. Indeed, any protective effects will only have an impact in the case of very long-term, regular endurance training.

The significant indirect cancer protection remains unaffected by the direct influence of regular endurance training on preventing malignancy.

Indirect cancer protection can be conferred, e. g., by reducing the proportion of body fat $(\checkmark$ Chapter 39$)$, improved diet, restraint in alcohol consumption or quitting smoking (Khan et al. 2010, Lee et Derakhshan 2013). 